ISSN 1995-5464 (Print); ISSN 2408-9524 (Online)

https://doi.org/10.16931/1995-5464.2021-4-120-125

Аневризмы селезеночной артерии

в сочетании с внепеченочной портальной

гипертензией при беременности

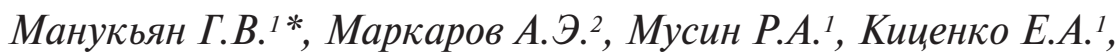
Апресян С.В. ${ }^{2}$, Лебезев В.М. ${ }^{1}$, Жигалова С.Б. ${ }^{1}$, Ризаева С.А. ${ }^{1}$, Щукина А.А. ${ }^{1}$

${ }^{\prime}$ Лаборатория экстренной хирургии и портальной гипертензии ГНЦ "Российский научный центр хирургии им. академика Б.В. Петровского”; 119991, Москва, ГСП-1, Абрикосовский пер., д. 2, Российская Федерация

${ }^{2}$ ГБУЗ “Городская клиническая больница им. А.К. Ерамишаниева ДЗМ”; 129327, Москва, ул. Ленская, д. 15, Российская Федерация

Клиническое наблюдение демонстрирует успешное лечение беременной с аневризмами селезеночной артерии больших размеров и угрозой их спонтанного разрыва. Беременность протекала на фоне наследственной тромбофилии, тромбоза и кавернозной трансформации воротной вены, внепеченочной портальной гипертензии и варикозных вен пищевода и желудка II-III степени. Выбранная хирургическая и акушерская тактика, хирургическое вмешательство, адекватная консервативная терапия и мультидисциплинарное наблюдение за течением беременности обеспечили успешное родоразрешение на 38-й неделе путем кесарева сечения, что позволило сохранить жизнь ребенку и матери.

Ключевые слова: аневризма селезеночной артерии, тромбофилия, тромбоз воротной вены, кавернозная трансформация, внепеченочная портальная гипертензия, беременность

Ссылка для цитирования: Манукьян Г.В., Маркаров А.Э., Мусин Р.А., Киценко Е.А., Апресян С.В., Лебезев В.М., Жигалова С.Б., Ризаева С.А., Щукина А.А. Аневризмы селезеночной артерии в сочетании с внепеченочной портальной гипертензией при беременности. Анналы хирургической гепатологии. 2021; 26 (4): 120-125.

https://doi.org/10.16931/1995-5464.2021-4-120-125.

Авторы подтверждают отсутствие конфликтов интересов.

\title{
Splenic artery aneurysms and extrahepatic portal hypertension during pregnancy
}

Manukyan G.V.I ${ }^{*}$, Markarov A.E. ${ }^{2}$, Musin R.A. ${ }^{1}$, Kitsenko E.A. ${ }^{1}$, Apresyan S.V.', Lebezev V.M. ${ }^{1}$, Ghigalova S.B. ${ }^{1}$, Rizaeva S.A. ${ }^{1}$, Shchukina A.A. ${ }^{1}$

${ }^{1}$ Laboratory of Emergency Surgery and Portal Hypertension SSC "Petrovsky Russian Research Center of Surgery"; 2, Abrikosovsky lane, Moscow, 119991, Russian Federation

${ }^{2}$ A.K. Eramishantsev City Clinical Hospital of the Department of Health of Moscow; 15, Lenskaya str., Moscow, 129327, Russian Federation

Clinical case demonstrates successful treatment of a pregnant woman, who had multiple giant splenic artery aneurysms with a high risk of spontaneous rupture. Pregnancy proceeded along with hereditary thrombophilia, thrombosis and cavernous transformation of the vena cava, extrahepatic portal hypertension, esophageal and gastric varices II-III. The surgical and obstetric tactics, surgical intervention, appropriate salvage and multidisciplinary pregnancy follow-up ensured a successful Cesarean delivery at 38 weeks, saving the life of the child and mother.

Keywords: splenic artery aneurysm, thrombophilia, portal vein thrombosis, cavernous transformation, portal hypertension, pregnancy

For citation: Manukyan G.V., Markarov A.E., Musin R.A., Kitsenko E.A., Apresyan S.V., Lebezev V.M., Ghigalova S.B., Rizaeva S.A., Shchukina A.A. Splenic artery aneurysms and extrahepatic portal hypertension during pregnancy. Annaly khirurgicheskoy gepatologii = Annals of HPB surgery. 2021; 26 (4): 120-125. (In Russian). https://doi.org/10.16931/1995-5464.20214-120-125.

There is no conflict of interests. 
Изолированная аневризма висцеральных артерий брюшной полости является достаточно редким наблюдением $[1,2]$. Аневризма селезеночной артерии (ACA) составляет $60 \%$ от их общего числа [2]. Чаще всего АCA оказывается случайной находкой при УЗИ, КТ или при развитии осложнения в виде разрыва [3-8]. Частота летального исхода при этом может достигать $25 \%$ без сопутствующей беременности $[2,7,8]$. В литературе отсутствуют сводные данные о распространенности ACA у беременных, а имеющиеся публикации основаны на отдельных наблюдениях. Тем не менее считают, что беременность тесно связана с формированием АCA $[7,9]$. Еще в 1942 г. было высказано предположение о том, что аневризматическая дилатация артерии является результатом двух факторов: слабости артериальной стенки и повышения в ней давления [10]. При беременности этому способствуют гормональные, ангиодиспластические изменения, гемодинамические и другие механизмы $[9,11]$. В числе гормональных влияний в первую очередь упоминают эстрогены, прогестерон и релаксин [2, 12-15]. Дополнительную нагрузку на артериальную стенку оказывают физиологические изменения, сопровождающие беременность, - усиление сердечного выброса и увеличение объема циркулирующей крови $[2,11,13]$. В течение беременности частота разрыва АСА увеличивается, при этом материнская смертность увеличивается до $70 \%[6,7,11]$, частота интранатальной гибели плода - до 90\% [16, 17].

Сочетание ACA с портальной гипертензией (ПГ) при беременности - дополнительный риск возможного разрыва аневризмы $[3,10,14,18$, 19]. Важнейшим фактором воздействия на АCA является блок воротного кровотока, препятствующий оттоку крови из селезенки и способствующий гипертензии в приносящем артериальном русле [3, 19-21].

Мнения специалистов о хирургической тактике ведения таких пациенток в плановой ситуации противоречивы. При аневризмах $>2$ см рекомендовано проводить лечение, при этом существуют наблюдения неосложненного течения гигантских ACA и в то же время описаны разрывы более мелких аневризм [22, 23]. При сочетании ACA и беременности большинство специалистов придерживаются активной тактики с применением различных эндоваскулярных и лапароскопических технологий, а также открытых вмешательств при вынашивании беременности [23-25].

Таким образом, сочетание беременности с АCА и ПГ сопровождается большой вероятностью фатального перинатального исхода у матери и плода. Приводим клиническое наблюдение.
Пациентка 24 лет госпитализирована 02.04.2019 с жалобами на периодическое чувство дискомфорта в левом подреберье. В 2017 г. срочные роды путем кесарева сечения. В 2018 г. обнаружены спленомегалия, тромбоцитопения и множественные АСА. Ангиохирургом рекомендовано динамическое наблюдение. В декабре 2018 г. диагностирована беременность, которую было предложено прервать, однако пациентка категорически отказалась. Повторно консультирована ангиохирургом, рекомендована операция после (!) вынашивания беременности. Осмотрена в НМИЦ гематологии по поводу тромбоцитопении, направлена в РНЦХ им. акад. Б.В. Петровского на базе ГКБ им. А.К. Ерамишанцева. Учитывая большой риск разрыва АCA, а также угрозу жизни матери и плода, госпитализирована в отделение экстренной хирургии и портальной гипертензии. Эритроциты 4,36 × 1012/л, гемоглобин 127 г/л, гематокритное число 38,7\%, тромбоцитов $101 \times 10^{9} / л$, лейкоцитов $7,1 \times 10^{9} /$ л, АЧТВ 29,1 с, протромбиновое время 13,4 с, протромбин $75 \%$, МНО 1,23, фибриноген 2,22 г/л. Выполнена МСКТ органов брюшной полости. Определена кавернозная трансформация воротной вены; селезеночная вена (СА) расширена, селезенка увеличена, $121 \times 71 \times 195$ мм; множество коллатералей до 9-13 мм (рис. 1). Поджелудочная железа деформирована, по верхнему краю на уровне тела и хвоста несколько мешотчатых аневризм СА $36 \times 51 \times 46,24 \times 30 \times 38$ и $13 \times 20 \times 22$ мм с кальцинатами в стенке (рис. 2). ЭхоКГ: сократительная способность миокарда левого желудочка в норме, ФВ 62\%. ЭГДС: в пищеводе 4 ствола варикозных вен (BВ) до 0,5 см, не напряжены, без васкулопатии, в желудке 3 ствола ВВ до 0,40,5 см. УЗИ матки и плода: фетометрические показатели соответствуют 17-18 нед беременности. Рубец на матке. Проведен междисциплинарный городской консилиум. Категорический отказ пациентки от прерывания беременности, большая вероятность разрыва ACA, отсутствие активности в течении тромбофилии, малая вероятность кровотечения из ВВ пищевода и желудка, а также нормальное течение беременности обусловили решение о необходимости оперировать больную, несмотря на имеющиеся риски. Ввиду топографо-анатомических особенностей АСА применение мини-инвазивных вмешательств было исключено. Объем операции - спленэктомия с резекцией аневризм селезеночной артерии. Основными задачами операции считали минимизацию травматичности вмешательства, предотвращение повреждения поджелудочной железы и интраоперационной кровопотери. 08.04.2019 под эндотрахеальным наркозом выполнена срединная лапаротомия. При ревизии селезенка значительно увеличена, множество расширенных венозных коллатералей портального бассейна. Желудок мобилизован по большой кривизне. Забрюшинно обнаружено напряженное пульсирующее образование $12 \times 8$ см, контурирующее по верхнему краю поджелудочной железы на уровне тела и хвоста, тесно прилегающее к железе, воротам селе- 


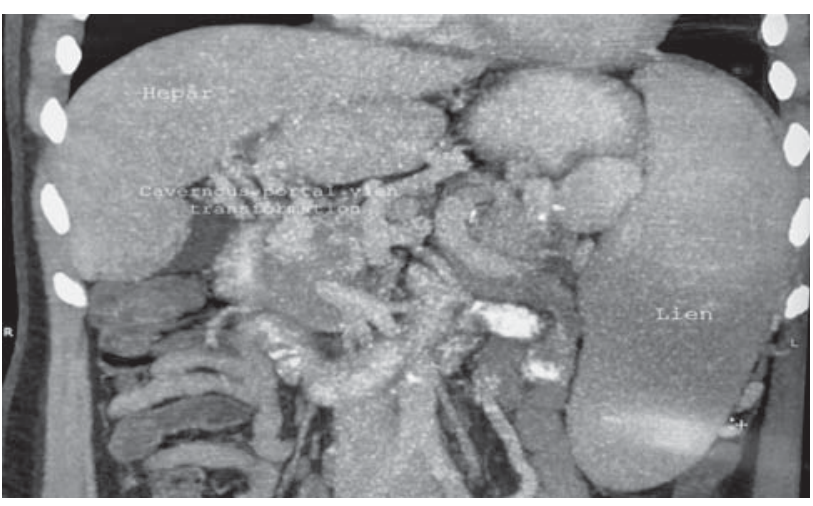

Рис. 1. Компьютерная томограмма. Кавернозная трансформация воротной вены, спленомегалия, множество спонтанных порто-портальных и порто-кавальных коллатералей.

Fig. 1. CT scans. Cavernous transformation of the vena cava, splenomegaly, multiple spontaneous porto-portal and portocaval collaterals.

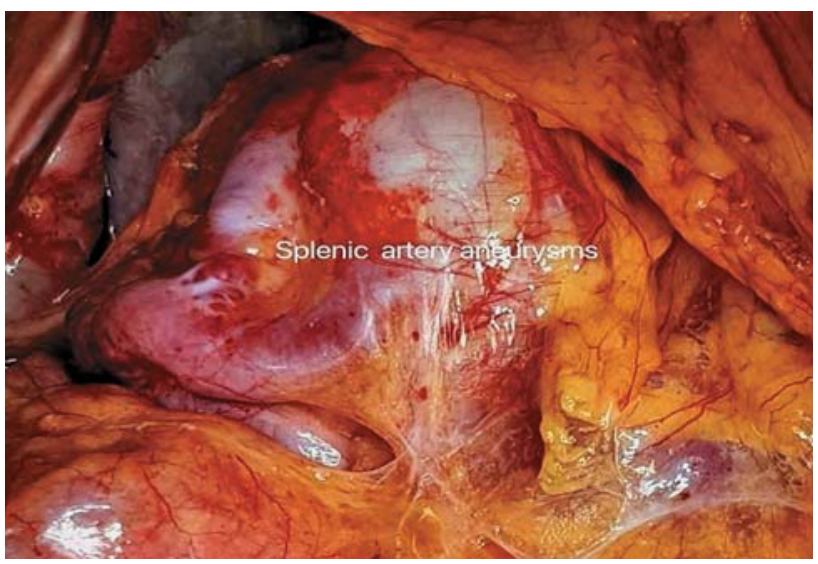

Рис. 3. Интраоперационное фото. Этап вскрытия сальниковой сумки. Виден конгломерат аневризм селезеночной артерии.

Fig. 3. Intraoperative photo. Access to the lesser sac. The conglomerate of splenic artery aneurysms.

зенки и диафрагме (рис. 3). Вскрыта брюшина, выделен сегмент СА проксимальнее пульсирующего образования, взят на турникет, пережат. При этом напряжение и пульсация образования значительно уменьшились, селезенка умеренно сократилась и стала мягче. Выполнена спленэктомия с последующей мобилизацией и удалением сосудистого конгломерата, образованного всеми аневризмами СА, единым блоком (рис. 4). Общая кровопотеря 250-300 мл. Манипуляций на ВВ не проводили, дабы не увеличивать объем и травматичность вмешательства. Кроме того, были основания ожидать, что после спленэктомии гипертензионная нагрузка на ВВ должна уменьшиться. Операция закончена дренированием левого поддиафрагмального пространства. Послеоперационный период протекал гладко, дренаж удален

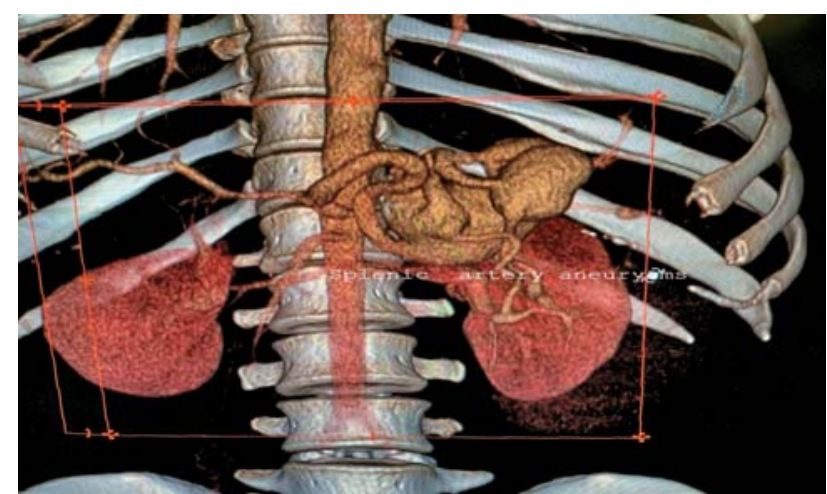

Рис. 2. Компьютерная томограмма, 3D-реконструкция. Аневризматические расширения селезеночной артерии.

Fig. 2. CT scans, 3D-reconstruction. Aneurysms of splenic artery.

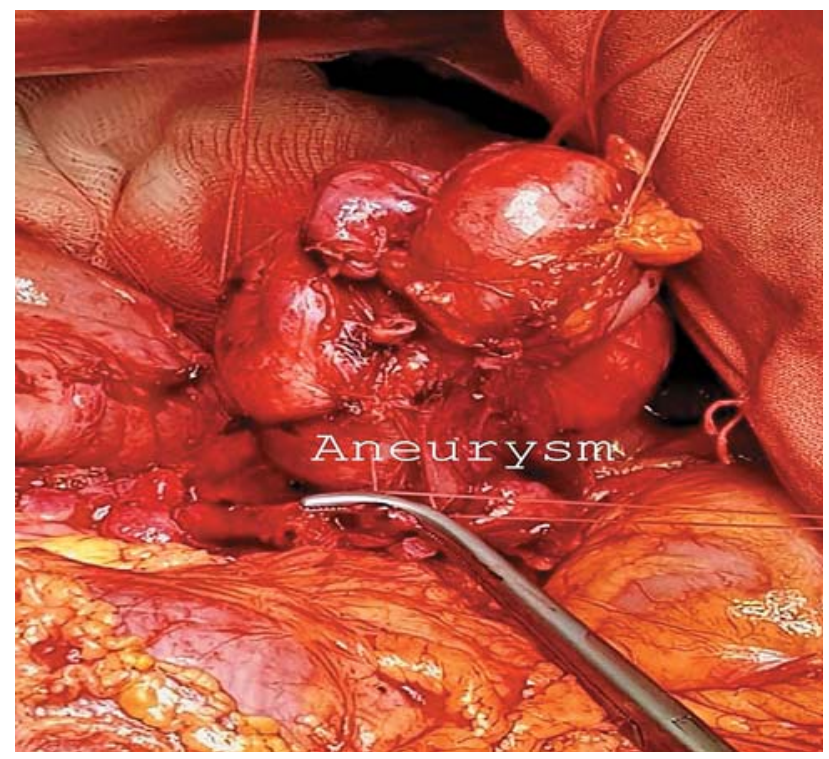

Рис. 4. Интраоперационное фото. Этап удаления аневризм селезеночной артерии единым блоком.

Fig. 4. Intraoperative photo. En-bloc resection of splenic artery aneurysms.

на 5-е сутки, заживление послеоперационной раны первичным натяжением. Выполнено гистологическое исследование. В селезенке атрофия лимфоидных фолликулов, обеднение клеточного состава красной пульпы, характерное для ПГ. Фрагменты артерии мышечно-эластического типа с истонченной эластической мембраной, наличием фиброзной бляшки с кальцинозом. Течение беременности - без осложнений. При ЭГДС отмечено уменьшение расширения BВ пищевода и желудка до I-II степени. Получала умеренную антикоагулянтную терапию под контролем коагулограммы. 05.09.2019 на 38-й неделе беременности выполнено плановое кесарево сечение. Родилась живая доношенная девочка: 2760 г, 52 см, 8-9 баллов по Апгар. Послеоперационное течение гладкое. Осмотрена через 6 и 12 мес после родов, со- 
стояние хорошее, жалоб нет, осложнений по течению тромбофилии и ПГ не отмечено. При ЭГДС ВВ пищевода и желудка без отрицательной динамики. Лабораторные показатели в пределах допустимых значений. Получает поддерживающую терапию: апиксабан 5 мг в сутки, фолиевую кислоту 400 мг в сутки и и витамины группы В 1 таб. в неделю.

Представленное клиническое наблюдение требует остановиться на следующих основных положениях. Наличие АСА при беременности является серьезным фактором риска, угрожающим жизни матери и плода. Сочетание с ПГ усугубляет течение аневризмы и делает сомнительным прогноз беременности без рассмотрения показаний к ликвидации угрозы со стороны АСА и ВВ пищевода и желудка. Выбор метода хирургического вмешательства следует основывать на оценке локализации и размеров АCA, ее синтопии с другими органами. Предпочтение следует отдавать мини-инвазивным, эндоваскулярным или лапароскопическим вмешательствам. При невозможности их применения следует выполнять открытую операцию. При сочетании беременности с АСА и ПГ требуется мультидисциплинарный подход - очень взвешенный экспертный анализ хирургами, акушерами, а также при необходимости гематологами, гепатологами и другими специалистами для выработки хирургической и акушерской тактики в отношении беременности.

\section{Участие авторов}

Манукьян Г.В. - полостное оперативное вмешательство, написание статьи.

Маркаров А.Э. - ассистенция в операции, участие в курации больной.

Мусин Р.А. - ассистенция в операции, оформление иллюстраций.

Киценко Е.А. - ассистенция в операции, подбор литературы.

Апресян С.В. - операция кесарева сечения, курация больной.

Лебезев В.М. - написание фрагмента статьи, участие в курации больной.

Жигалова С.Б. - обеспечение эндоскопического контроля, участие в курации больной.

Ризаева С.А. - ассистенция в операции, лечащий врач.

Щукина А.А. - курация больной, подбор литературы.

\section{Authors participation}

Manukyan G.V. - cavitary surgical intervention, writing the article.

Markarov A.E. - assistance in surgical intervention, participation in patient follow-up.

Musin R.A. - assistance in surgical intervention, selection and design of illustrations.

Kitsenko E.A. - assistance in surgical intervention, literature review.
Apresyan S.V. - cesarean section, patient follow-up.

Lebezev V.M. - writing a fragment of the article, participation in patient follow-up.

Zhigalova S.B. - endoscopic control and treatment, participation in patient follow-up.

Rizaeva S.A. - assistance in surgical intervention, attending physician.

Shchukina A.A. - patient follow-up, selection of literature.

\section{Список литературы [References]}

1. Jana M., Gamanagatti S., Mukund A., Paul S., Gupta P., Garg P., Chattopadhyay T., Sahni P. Endovascular management in abdominal visceral arterial aneurysms: a pictorial essay. World J. Radiol. 2011; 3 (7): 182-187.

http://doi.org/10.4329/wjr.v3.i7.182

2. Sadat U., Dar O., Walsh S., Varty K. Splenic artery aneurysms in pregnancy - a systematic review. Int. J. Surg. 2008; 6 (3): 261-265. http://doi.org/10.1016/j.ijsu.2007.08.002

3. Morton A. Splenic artery aneurysms, portal hypertension and pregnancy. J. Hepatol. 2019; 70 (5): 1025-1026.

http://doi.org/10.1016/j.jhep.2018.12.019

4. Wiener Y., Tomashev R., Neeman O., Itzhakov Z., Heldenberg E., Melcer Y., Maymon R. Splenic artery aneurysms during pregnancy: an obstetric nightmare. Obstet. Gynecol. Reprod. Biol. 2019; 237: 121-125. http://doi.org/10.1016/j.ejogrb.2019.04.029

5. Parrish J., Maxwell C., Beecroft J.R.J. Splenic artery aneurysm in pregnancy. Obstet. Gynaecol. Can. 2015; 37 (9): 816-818. http://doi.org/10.1016/S1701-2163[15]30153-5

6. Jackson H.T., Diaconu S.C., Maluso P.J., Abell B., Lee J. Ruptured splenic artery aneurysms and the use of an adapted fast protocol in reproductive age women with hemodynamic collapse: case series. Case Rep. Emerg. Med. 2014; 2014: 454923. http://doi.org/10.1155/2014/454923

7. Ballout R.A., Ghanem R., Nassar A., Hallal A.H., Ghulmiyyah L.M.J. Splenic artery aneurysm [SAA] rupture in pregnancy: a case report of a rare but life-threatening obstetrical complication. Womens Health Dev. 2019; 2 (1): 19-27. http://doi.org/10.26502/fjwhd.2644-2884004

8. Jacobson J., Gorbatkin C., Good S., Sullivan S. Splenic artery aneurysm rupture in pregnancy. Am. J. Emerg. Med. 2017; 35 (6): 935.e5-935.e8. http://doi.org/10.1016/j.ajem.2016.12.035

9. Aubrey-Bassler F.K., Sowers N. 613 cases of splenic rupture without risk factors or previously diagnosed disease: a systematic review. BMC Emerg. Med. 2012; 12: 11.

http://doi.org/10.1186/1471-227X-12-11

10. Trimble W.K., Hill J.H. Congestive splenomegaly [Banti's disease] due to portal stenosis without hepatic cirrhosis; aneurysms of the splenic artery. Arch. Pathol. Lab. Med. 1942; 34: 423.

11. Veluppillai C., Perreve S., de Kerviler B., Ducarme G. Splenic arterial aneurysm and pregnancy: a review. Presse Med. 2015; 44 (10): 991-994. http://doi.org/10.1016/j.lpm.2015.06.009

12. Veterano C., Monteiro E., Rego D., Soares P., Almeida P., Almeida R. Laparoscopic resection of a splenic artery aneurysm with vascular reconstruction during pregnancy. Ann. Vasc. Surg. 2021; 72: 666.e7-666.e11. Epub 2020 Nov 21. http://doi.org/10.1016/j.avsg.2020.10.016

13. Andrade F., Shukla A., Bureau C., Senzolo M., D'Alteroche L., Heurgué A., Turon F., Garcia-Pagan J.C., Oberti F., Tripathi D., Rautou P.E.J. Reply to: "Splenic artery aneurysms, portal hypertension and pregnancy". J. Hepatol. 2019; 70 (5): 1026-1027. http://doi.org/10.1016/j.jhep.2019.01.006. 
14. Hoekstra J., Seijo S., Rautou P.E., Janssen H.L.A., Valla D.C., Plessier A. Pregnancy in women with portal vein thrombosis: results of a multicentric European study on maternal and fetal management and outcome. J. Hepatol. 2012; 57 (6): 1214-1219. http://doi.org/10.1016/j.jhep.2012.07.034

15. d'Alteroche L., Perarnau J.-M., Perrotin F., Bacq Y. Portal hypertension and pregnancy. Gastroenterol. Clin. Biol. 2008; 32 (5Pt1): 541-546. http://doi.org/10.1016/j.gcb.2008.02.028

16. Chaichian S., Mehdizadeh A., Akbarian A., Groohi B., Hanahmadi N., Alaghehbandan R. Rupture of splenic artery aneurysm with portal hypertension during pregnancy: a case report. $J$. Obstet. Gynaecol. Can. 2006; 28 (4): 303-304. PMID: 16776908

17. Samberkar P.N., Chow T.K., Samberkar S.P. Fatal spontaneous rupture of splenic artery aneurysm in third trimester pregnancy. Malays. J. Pathol. 2018; 40 (3): 337-341. PMID: 30580366

18. Aroor A.R., Prakasha S.R., Attar N.R. Multiple splenic artery aneurysms: a rare cause of extrahepatic portal hypertension and massive splenomegaly. J. Clin. Diagn. Res. 2014; 8 (9): MD01-2. http://doi.org/10.7860/JCDR/2014/8498.4821

19. Subbaiah M., Kumar S., Roy K.K., Sharma J.B., Singh N. Extrahepatic portal-vein obstruction in pregnancy. Taiwan $J$. Obstet. Gynecol. 2015; 54 (4): 394-397. http://doi.org/10.1016j.tjog.2013.11.0912

20. Beksac K., Karakoc D. Multiple giant splenic artery aneurisms causing sinistral [left-sided] portal hypertension. Case Rep. Gatrointest. Med. 2016: 6278452. http://doi.org/10.1155/2016/6278452
21. Rehman Z.U. Multiple giant splenic artery aneurisms with hypersplenism and portal hypertension: a case report. Ann. Vasc. Dis. 2019; 12 (2): 250-252. http://doi:/10.3400/avd.cr.19-00021

22. Selo-Ojeme D.O., Welch C.C. Review: spontaneous rupture of splenic artery aneurysm in pregnancy. Eur. J. Obstet. Gynecol. Reprod. Biol. 2003 15; 109 (2): 124-127. http:/doi.org/10.1016/s0301-2115(03)00094-0

23. Samamé J., Kaul A., Garza U., Echeverria A., Galvani C. Laparoscopic aneurysm resection and splenectomy for splenic artery aneurysm in the third trimester of pregnancy. Surg. Endosc. 2013; 27 (8): 2988-2991. http://doi.org/10.1007/s00464-013-2822-X

24. Tlili A., Trigui A., Dkhil O., Feki W., Rejab H., Ameur H.B., Boujelbene S., Mnif Z. Splenic artery aneurysm rupture at the end of pregnancy: a case study. Pan. Afr. Med. J. 2019; 34: 63. http://doi.org/10.11604/pamj.2019.34.63.18598

25. Лищенко А.Н., Анисимов С.Н., Вартанян С.М., Бочкарева И.В., Шалагинов С.И., Шалагинова М.Г., Андреева М.Б., Воропаева Ю.А. Спонтанный разрыв истинной аневризмы селезеночной артерии у беременных. Вестник хирургии им. И.И. Грекова. 2015; 174 (4): 84-86. https://doi.org/10.24884/0042-4625-2015-174-4

Lishchenko A.N., Anisimov S.N., Vartanyan S.M., Bochkareva I.V., Shalaginov S.I., Shalaginova M.G., Andreeva M.B., Voropaeva Yu.A. Spontaneous splenic artery aneurysm rupture in pregnancy. Grekov's Bulletin of Surgery. 2015; 174 (4): 84-86. https://doi.org/10.24884/0042-4625-2015-174-4 (In Russian)

\section{Сведения об авторах [Authors info]}

Манукьян Гарик Ваганович - доктор мед. наук, заведующий отделением экстренной хирургии и портальной гипертензии ГНЦ РНЦХ им. акад. Б.В. Петровского. https://orcid.org/0000-0001-8064-1964. E-mail: drmanukyan@mail.ru

Маркаров Арнольд Эдуардович - канд. мед. наук, заместитель главного врача по хирургической помощи ГБУз ГКБ им. А.К. Ерамишанцева ДЗМ. https://orcid.org/0000-0002-0392-8280. E-mail: markarnold@mail.ru

Мусин Рустам Абузарович - канд. мед. наук, старший научный сотрудник отделения экстренной хирургии и портальной гипертензии ГНЦ РНЦХ им. акад. Б.В. Петровского. https://orcid.org/0000-0002-3380-2224. E-mail: surgery@mail.ru Киценко Евгений Александрович - доктор мед. наук, ведущий научный сотрудник отделения экстренной хирургии и портальной гипертензии ГНЦ РНЦХ им. акад. Б.В. Петровского. https://orcid.org/0000-0002-8268-3129. E-mail: kitsenko-surgeon@mail.ru

Апресян Сергей Владиславович - доктор мед. наук, заместитель главного врача по акушерско-гинекологической помощи ГБУЗ ГКБ им. А.К. Ерамишанцева ДЗМ. https://orcid.org/0000-0002-7310-974X. E-mail: sapresyan@mail.ru

Лебезев Виктор Михайлович - доктор мед. наук, главный научный сотрудник отделения экстренной хирургии и портальной гипертензии ГНЦ РНЦХ им. акад. Б.В. Петровского. https://orcid.org/0000-0002-0905-8941.

E-mail: viktorlebezev@yandex.ru

Жигалова Светлана Борисовна - доктор мед. наук, ведущий научный сотрудник отделения экстренной хирургии и портальной гипертензии ГНЦ РНЦХ им. акад. Б.В. Петровского. https://orcid.org/0000-0003-1006-3462. E-mail: zhigalova06@mail.ru

Ризаева Саида Алтынбайевна - аспирант отделения экстренной хирургии и портальной гипертензии ГНЦ РНЦХ им. акад. Б.В. Петровского. https://orcid.org/0000-0002-6815-6672. E-mail: cauduk@yandex.ru

Щукина Анастасия Андреевна - аспирант лаборатории экстренной хирургии и портальной гипертензии ГНЦ РНЦХ им. акад. Б.В. Петровского. https://orcid.org/0000-0001-9349-8327. E-mail: nastia3001@mail.ru

Для корреспонденции *: Манукьян Гарик Ваганович - 119991, Москва, ГСП-1, Абрикосовский пер., д. 2, лаборатория экстренной хирургии и портальной гипертензии ГНЦ РНЦХ им. акад. Б.В. Петровского, Российская Федерация. Тел.: +7-929-509-03-03. E-mail: drmanukyan@mail.ru 
Garik V. Manukyan - Doct. of Sci. (Med.), Head of the Department of Emergency Surgery and Portal Hypertension, Petrovsky Russian Research Center of Surgery. https://orcid.org/0000-0001-8064-1964. E-mail: drmanukyan@mail.ru

Arnold E. Markarov - Cand. of Sci. (Med.), Deputy Chief Physician for Surgical Treatment, A.K. Eramishantsev City Clinical Hospital of the Department of Health of Moscow. https://orcid.org/0000-0002-0392-8280. E-mail: markarnold@mail.ru

Rustam A. Musin - Cand. of Sci. (Med.), Senior Researcher of the Department of Emergency Surgery and Portal Hypertension, Petrovsky Russian Research Center of Surgery. https://orcid.org/0000-0002-3380-2224. E-mail: surgery@mail.ru

Evgeniy A. Kitsenko - Doct. of Sci. (Med.), Leading Researcher of the Department of Emergency Surgery and Portal Hypertension, Petrovsky Russian Research Center of Surgery. https://orcid.org/0000-0002-8268-3129. E-mail: kitsenko-surgeon@mail.ru

Sergey V. Apresyan - Doct. of Sci. (Med.), Deputy Chief Physician for Obstetric and Gynecological Care, A.K. Eramishantsev City Clinical Hospital of the Department of Health of Moscow. https://orcid.org/0000-0002-7310-974X. E-mail: sapresyan@mail.ru

Victor M. Lebezev - Doct. of Sci. (Med.), Chief Researcher of the Department of Emergency Surgery and Portal Hypertension, Petrovsky Russian Research Center of Surgery. https://orcid.org/0000-0002-0905-8941. E-mail: viktorlebezev@yandex.ru

Svetlana B. Zhigalova - Doct. of Sci. (Med.), Leading Researcher of the Department of Emergency Surgery and Portal Hypertension, Petrovsky Russian Research Center of Surgery. https://orcid.org/0000-0003-1006-3462. E-mail: zhigalova06@mail.ru

Saida A. Rizaeva - Post-graduate Student of the Department of Emergency Surgery and Portal Hypertension, Petrovsky Russian Research Center of Surgery. https://orcid.org/0000-0002-6815-6672. E-mail: cauduk@yandex.ru

Anastasia A. Shchukina - Post-graduate Student of the Laboratory of Emergency Surgery and Portal Hypertension, Petrovsky Russian Research Center of Surgery. https://orcid.org/0000-0001-9349-8327. E-mail: nastia3001@mail.ru

For correspondence*: Garik V. Manukyan - 2, Abrikosovsky lane, Moscow, 119991, Russia, Laboratory of Emergency Surgery and Portal Hypertension, Petrovsky Russian Research Center of Surgery, Russia. Phone: 8-929-509-03-03. E-mail: drmanukyan@mail.ru

Статья поступила в редакцию журнала 30.01.2021.

Received 30 January 2021.
Принята к публикации 28.09.21. Accepted for publication 28 September 2021. 